\title{
A ground-glass opacity increasing in size over eight years diagnosed as lung adenocarcinoma: A case report
}

\author{
Dian Ren*, Ming Dong*, Zuoqing Song, Jinghao Liu, Renwang Liu, hongyu Liu and Jun Chen* \\ Department of Lung Cancer Surgery, Tianjin Lung Cancer Institute; Tianjin Medical University, General Hospital; Heping District, Tianjin 300052, China \\ \#These authors contributed equally to this work
}

\begin{abstract}
A 62year-old man with history of pulmonary tuberculosis was found upon CT scan to have a ground-glassopacity (GGO) in the right lung. Given the patient's background, his primary doctor recommended yearly CT scan to follow the lesion. The lesion increased approximately $1 \mathrm{~cm}$ in size over the next eight years, and the patient underwent a video-assisted thoracoscopic right lower lobe resection. Pathologic examination revealed invasive pulmonary adenocarcinoma with lymph node metastasis. This indicates that, even if it grows slowly and is less than $2 \mathrm{~cm}$ in size, GGO may be malignant and associated with lymph node metastasis.
\end{abstract}

\section{Introduction}

Small pulmonary nodules (SPN) have become an increasingly important clinical issue over recent years given the increase in lung cancer worldwide and the increased discovery of nodules, as more patients are willing to undergo check-ups. There are many different causes of SPN, including precancerous lesions, tumor, inflammation, trauma, and vasculitis. SPN are divided into three types: pure GGO, hybrid GGO, and solid nodules; each type has unique clinical and epidemiological characteristics as well as different prognoses, prompting debate among pulmonary clinicians as to management.

\section{Case report}

A 62-year-old man with history of treated and stable pulmonary tuberculosis for the past 50 years underwent routine physical examination; a CT scan showed an abnormal lesion [ground-glass opacity (GGO)] in the lower lobe of right lung field. His past medical history also included a 20-year history of smoking, 20 years of hypertension, and 6 years of coronary heart disease. Considering his history of pulmonary tuberculosis and presence of GGO on CT, his doctor recommended annual follow-up, and he proceeded to have yearly CT scan over the next eight years. The GGO was initially stable, and then the entire size and the size of the solid part of the GGO both increased. Eventually the patient was referred to our hospital for diagnosis and further treatment of a persistent asymptomatic pulmonary nodule/GGO. The GGO caused no symptoms, grew from $1 \mathrm{~cm}$ to $2 \mathrm{~cm}$ during the eight-year follow-up period, appeared to invade the pleura, and had a predominantly solid appearance. Upon evaluation, the squamous cell carcinoma antigen (SCCA) level was $1.7 \mu \mathrm{g} / \mathrm{ml}$ (normal $<1.5 \mu \mathrm{g} / \mathrm{ml})$ before and after surgery and then normalized $(0.6 \mu \mathrm{g} / \mathrm{ml})$ after the first round of chemotherapy. The level of carcinoembryonic antigen, neuron-specific enolase, and cytokerantin-19-fragment (CYFRA21-1) were all-normal on first testing. Upon initial evaluation by a thoracic surgeon, the nodule appeared suspicious for malignancy given the appearance of the GGO and the increase in size; the surgeon gave the patient the options of early surgical resection versus ongoing observation. Based on personal preference, the patient was firstly performed the wedge resection of the lesion, and then underwent a lobectomy of the right lower lobe under video-assisted thoracoscopic surgery after diagnosis of carcinoma by intraoperative frozen section in December 2014. Pathological examination confirmed that the lesion was invasive pulmonary adenocarcinoma with lymph node (LN) metastasis (Groups 4 and 11). The patient electively refused genetic testing and underwent one round of chemotherapy, standard regimen (Pemetrexed $0.845 \mathrm{~g} x$ one day, cis-platinum $40 \mathrm{mg}$ x three days). The patient returned to the hospital for follow-up in September 2015; no progression was found (Figures 1 and 2).

\section{Discussion}

Pulmonary adenocarcinoma, like pulmonary tuberculosis, can appear as GGO, but the two differ in behavior. The initial diagnosis depends on characteristics on CT. Early stages of lung adenocarcinoma can appear as peripheral and solid lesions, with lobulation, spiculation, pleural indentation, and vascular convergence, but GGO signs are unusual [1]. Pure GGO may indicate a precancerous lesion. Malignant lesions typically develop rapidly, with short doubling time, while benign lesions usually remain stable. A greater proportion of GGO and bigger solid component in lesions can be indicative of malignancy $[2,3]$. Size of the solid component of the tumor in the mediastinal window visualized on high-resolution CT (HRCT) predicts higher risk and better prognosis in pathologically high-grade malignant lung adenocarcinoma, and these measures are more valuable than whole tumor size. The GGO component has been shown to be irrelevant in

Correspondence to: Jun Chen, Department of Lung Cancer Surgery, Tianjin Lung Cancer Institute; Tianjin Medical University, General Hospital; Heping District, Tianjin 300052, China, Tel: 86-22-60814844; E-mail: huntercj2004@yahoo.com

Keywords: ground-glass opacity, adenocarcinoma, lymph node metastasis, pulmonary tuberculosis

Received: September 28, 2015; Accepted: October 19, 2015; Published: October 22,2015 

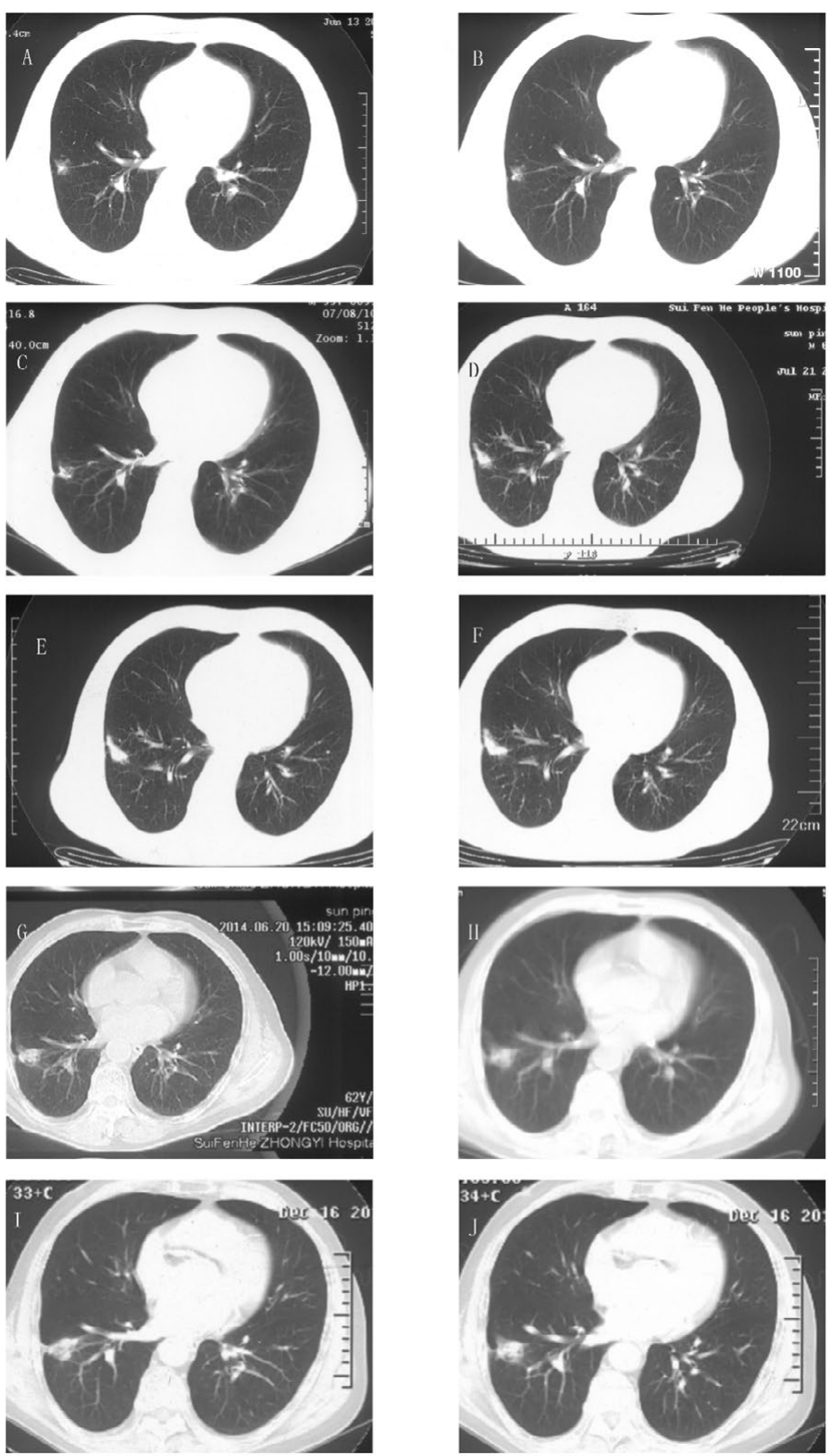

Figure 1. The images of chest CT scans during the eight-year follow-up period. CT scans on June 13, 2006 (A), July 16, 2007 (B), July 8, 2010 (C), July 21, 2011 (D), July 24, 2012 (E), August 14, 2013 (F), June 20, 2014 (G), September 30, 2014 (H) and December 16, 2014 (I-J). The CT scans showed a GGO in the lower lobe of the right lung upon initial diagnosis. The GGO grew very slowly over the next eight years; despite this, and considering the patient's medical history of pulmonary tuberculosis, it was recommended that the patient undergo annual follow-up.

terms of recurrence, while the $\mathrm{T}$ factor of the solid component may be a better parameter of prognosis [4].

The predilection sites of pulmonary tuberculosis in the lung include the apicoposterior and anterior segments of the superior lobe and dorsal and basal segments of the lower lobe. Tuberculosis lesions are mostly multiple, tree-in-bud, consolidated, and have different manifestations at different stages in the disease's development.

Although clinical decisions are made both by the physician as well as the patient and depend on each patient's psychological state and acceptance regarding surgery, the clinician's professional knowledge ultimately plays a leading role in deciding on management and treatment of GGO. The goal is to maximize the patient's survival time with the least amount of trauma.
Peter J. Sloane et al. summarized the diagnosis and management of pulmonary nodules according to the work of Gould et al. [5]. As the table demonstrates, annual follow-up is necessary for GGO lesions except for those less than $5 \mathrm{~mm}$ in size (Table 1). PET/CT is considered for solid nodules $4-6 \mathrm{~mm}$ in size but is not recommended to characterize the nodule.

There is controversy over the timing of surgery for GGOs that are highly suspected to be malignant. Some clinicians recommend follow-up until the nodule grows to over $3 \mathrm{~mm}$ in size or shows notable malignant features, such as significant shortening in the doubling time or if the proportion of GGO becomes less than $50 \%$. Some argue that surgery should be performed immediately when the GGO shows malignant features on radiographic imaging in case of the presence of pulmonary 


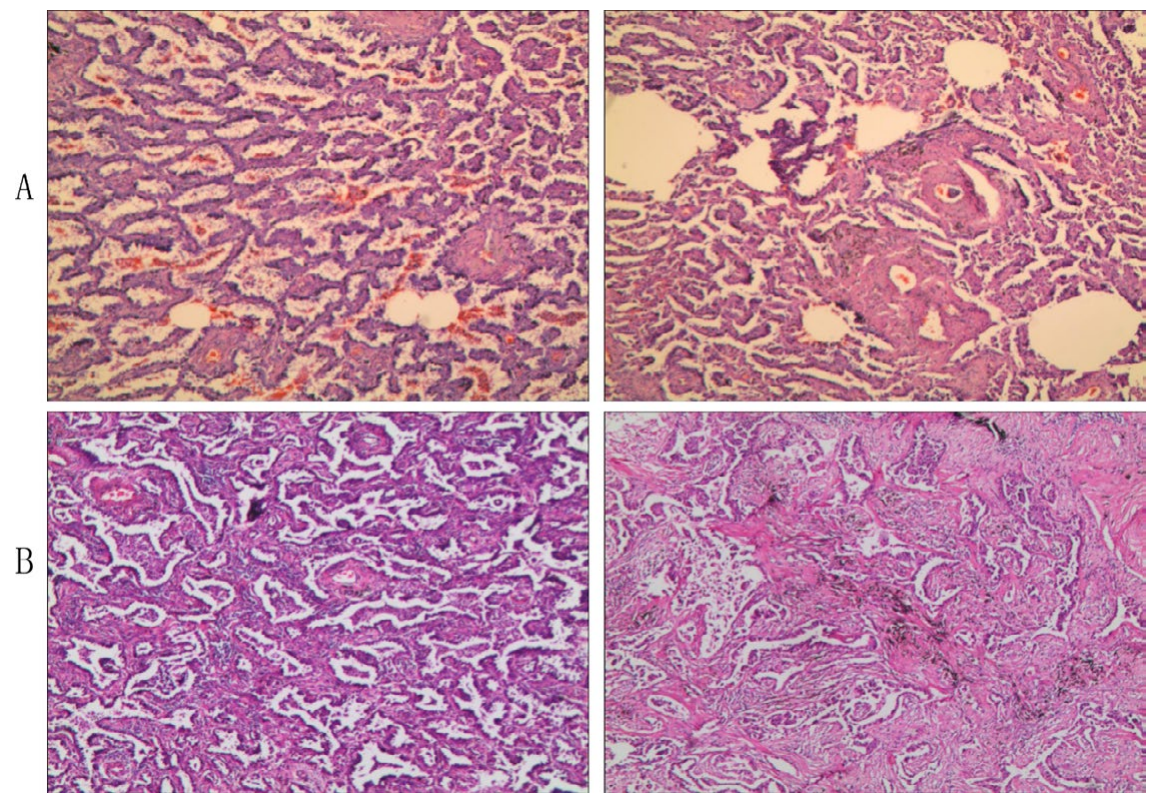

Figure 2. Pathologic analysis. (A) Intraoperative frozen section; (B) Paraffin section. It showed a nodule $1.1 \mathrm{~cm}$ in diameter that was determined to be invasive adenocarcinoma with acinar predominant and partial papillary predominant by H\&E staining. The bronchial stump is clean, and no metastatic carcinoma was found in lymph nodes from nearby bronchi or other lymph nodes (2, 3, 7, and 10 Group) except Groups 4 and 11, which had metastatic carcinoma.

Table 1. Guidelines for evaluation of pulmonary nodules ${ }^{\mathrm{a}}$

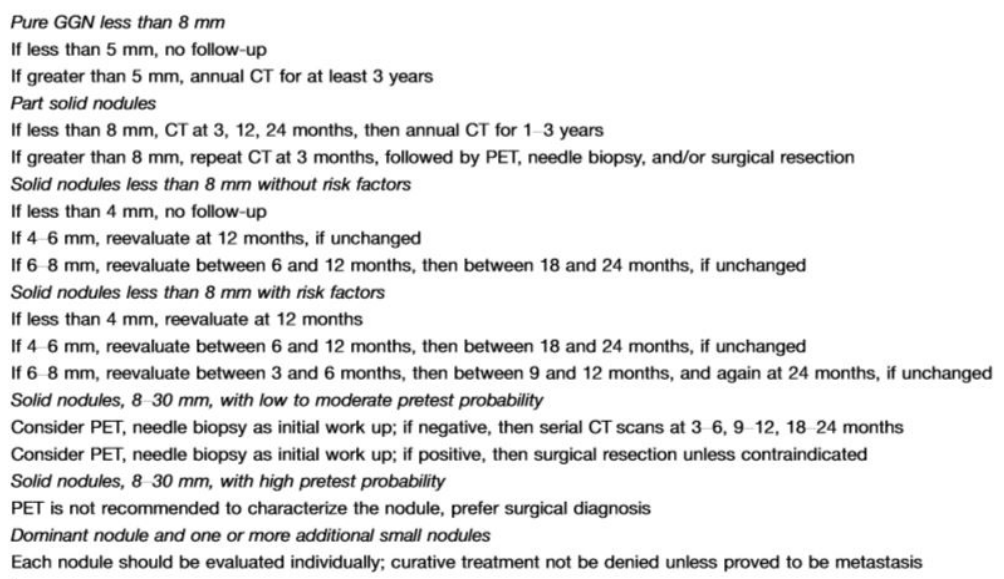

'Table summarizes diagnosis and management of pulmonary nodules as described by Gould et al. (1).

adenocarcinoma, which can have early oncogenic transformation or lymph node metastasis. Some theories argue that unnecessary lung resection can cause great trauma and suffering for the patient and is irreversible, however one must also consider the risk of missing the window of completely resecting pulmonary adenocarcinoma, as was revealed in our case $[6,7]$.

Controversy also exists regarding surgical treatment. In recent years, video-assisted thoracic surgery has been shown to have equivalent clinical value as thoracotomy for the treatment of lung cancer. One study showed that there was no difference in recurrence-free survival (RFS) between patients who underwent lobectomy, segmentectomy, or wedge resection for early stage lung cancer [8]; furthermore, there is no evidence that shows that segmentectomy is superior to lobectomy or wedge resection $[9,10]$. For most cases of GGO, wedge resection may be the best choice given that it is least invasive.
Even though lymph node metastasis occurs in only a small number of patients with early-stage lung cancer, systematic lymph node dissection is necessary for a small percentage of patients. Thus, it important to evaluate when lymph node dissection should be performed in all patients with clinical stage IA lung adenocarcinoma. According to research by Park SI et al., there is little to no additional benefit of mediastinal LN dissection for patients with pure GGNs [11]. According to $\mathrm{Hu} \mathrm{DZ}$ et al.'s report, patients with clinical stage IA lung adenocarcinoma who underwent surgical resection and had an intraoperative diagnosis could benefit from systematic lymph node dissection; these findings were especially true for patients with acarcinoembryonic antigen level exceeding $5 \mathrm{ng} / \mathrm{dL}$ and a maximum standardized uptake value exceeding 5 [12].

It is difficult to determine the RFS of GGO-dominant tumors that are defined as stage IA adenocarcinoma, as these cases typically do 
not undergo surgery. Although not currently recommended because research on it is divided, radiologic, noninvasive evaluation using the maximum standardized uptake value of 18 -fluorodeoxyglucose on $\mathrm{PET} / \mathrm{CT}(\mathrm{C} / \mathrm{T}$ ratio $</=25)$ may be a good indicator for surgery $[8,13]$. The proportion of the solid part in GGO-dominant tumors has also been considered to be a good indicator for surgery, because studies have shown correlation between higher percentage of the solid part of the tumor and increased aggressiveness in the tumor's behavior [14].

\section{Acknowledgements}

This work was supported by grants from the National Natural Science Foundation of China $(81172233,81372306)$, the Specialized Research Fund for the Doctoral Program of Higher Education (20131202110004), the Tianjin Natural Science Foundation (13JCYBJC22600). Funding sources had no role in study design, data collection, and analysis; in the decision to publish; or in the preparation of the manuscript.

\section{Competing interests}

The authors have declared that no competing interests exist.

\section{Authors contributions}

DR and MD collected all data and authored the manuscript. ZQS, JHL, and RWL were responsible for patient care and analysis of follow-up data. HYL analyzed the data and provided histopathologic confirmation. JC performed the surgical procedure, and also contributed to data analysis and shaping of the manuscript. All authors have read and approved the final manuscript.

\section{References}

1. Ren J, Zhou J, Ding W, Zhong B, Zhou J2 (2014) Clinicopathological characteristics and imaging features of pulmonary adenocarcinoma with micropapillary pattern. Zhonghua Zhong Liu Za Zhi 36: 282-286. [Crossref]

2. Ge X, Gao F, Li M, Chen Y, Lü F, et al. (2014) Diagnostic value of solid component for lung adenocarcinoma shown as ground-glass nodule on computed tomography. Zhonghua Yi Xue Za Zhi 94: 1010-1013. [Crossref]

3. Yamazaki M, Ishikawa H, Kunii R, Tasaki A, Sato S, et al. (2014) Relationship between CT features and high preoperative serum carcinoembryonic antigen levels in early-stage lung adenocarcinoma. Clin Radiol 69: 559-566. [Crossref]

4. Murakawa T, Konoeda C, Ito T, Inoue Y, Sano A, et al. (2013) The ground glass opacity component can be eliminated from the T-factor assessment of lung adenocarcinoma Eur J Cardiothorac Surg 43: 925-932. [Crossref]

5. Jin N, Sloane PJ (2014) Evaluation of pure ground glass pulmonary nodule: a case report. J Community Hosp Intern Med Perspect 4.[Crossref]

6. Seok Y, Cho S, Kim K, Jheon S (2014) Partly solid pulmonary nodules: waiting for change or surgery outright? Interact Cardiovasc Thorac Surg 19: 556-560. [Crossref]

7. Heo EY, Lee KW, Jheon S, Lee JH, Lee CT, et al. (2011) Surgical resection of highly suspicious pulmonary nodules without a tissue diagnosis. Jpn J Clin Oncol 41: 1017 1022. [Crossref]

8. Tsutani Y, Miyata Y, Nakayama H, Okumura S, Adachi S, et al. (2014) Appropriatesublobar resection choice for ground glass opacity-dominant clinical stage IA lung adenocarcinoma: wedge resection or segmentectomy. Chest 145: 66-71. [Crossref]

9. Bao F, Ye P, Yang Y, Wang L, Zhang C, et al. (2014) Segmentectomy or lobectomy for early stage lung cancer: a meta-analysis. Eur J Cardiothorac Surg 46: 1-7. [Crossref]

10. Landreneau RJ, Normolle DP, Christie NA, Awais O, Wizorek JJ, et al. (2014) Recurrence and survival outcomes after anatomic segmentectomy versus lobectomy for clinical stage I non-small-cell lung cancer: a propensity-matched analysis. $J$ Clin Oncol 32: 2449-2455. [Crossref]

11. Sim HJ, Choi SH, Chae EJ, Kim HR, Kim YH, et al. (2014) Surgical management of pulmonary adenocarcinoma presenting as a pure ground-glass nodule. Eur $J$ Cardiothorac Surg 46: 632-636. [Crossref]

12. Ye B, Cheng M, Li W, Ge XX, GengJF, et al. (2014) Predictive factors for lymph node metastasis in clinical stage IA lung adenocarcinoma. Ann Thorac Surg 98: 217-223. [Crossref]

13. Cho JH, Choi YS, Kim J, Kim HK, Zo JI, et al. (2015) Long-term outcomes of wedge resection for pulmonary ground-glass opacity nodules. Ann Thorac Surg 99: 218-222. [Crossref]

14. Nakamura S, Fukui T, Kawaguchi K, Fukumoto K, Hirakawa A, et al. (2015) Does ground glass opacity-dominant feature have a prognostic significance even in clinical T2aN0M0 lung adenocarcinoma? Lung Cancer 89: 38-42. [Crossref]

Copyright: (C2015 Ren D. This is an open-access article distributed under the terms of the Creative Commons Attribution License, which permits unrestricted use, distribution, and reproduction in any medium, provided the original author and source are credited. 